\title{
EL CONSUMO DE BIENES RELIGIOSOS Una introducción económica al caso español
}

\author{
PABLO BRAÑAS-GARZA* \\ Universidad de Jaén
}

\author{
PALABRAS CLAVE ADICIONALES \\ Comportamiento del consumidor, Religión, \\ Asistencia a la iglesia, Católicos españoles.
}

\section{ADDITIONAL KEYWORDS}

Consumer Behaviour, Religion, Church-atten-

dance, Spanish Roman Catholics.

RESUMEN. Este trabajo explora el comportamiento religioso de los españoles desde el enfoque de la Economía de la Religión. En un marco microeconómico y utilizando los argumentos de AzziEhrenberg (1975) —-motivo salvación, consumo y profesional-, la práctica religiosa resulta racional. En esta investigación estudiamos si estas hipótesis pueden explicar el comportamiento religioso de los españoles. Para ello se realizan análisis descriptivos y multivariantes que nos permiten concluir que: los modelos teóricos explican bien los datos españoles; que el sexo, la edad, el tamaño de la familia y la educación son determinantes positivos de la asistencia a iglesia; que el ejemplo de los padres influye en la actividad religiosa actual de los hijos.

ABSTRACT. This paper uses the Economic of Religion issues to explore Spanish religious behaviour. Under this microeconomic framework religious practise is considered "rational" using AzziEhrenberg (1975) arguments: salvation, social and professional motives. We test if that hypotheses may explain Spanish religious activity. To this end we run descriptive and multivariate analysis that allows us to conclude the folllowing: theoretical models well perform Spanish data; gender, ageing, family size and education are positive determinant of church-attendance; the influence of parents behaviour on children religious activity.

- Parte de este trabajo ha sido previamente presentado en IZA (Bonn, 2002) y en el Workshop de Microeconomía de CentrA (Sevilla, 2002). Agradezco especialmente a Javier Rodero (CentrA) y a Shoshana Neuman (BIU y CEPR) sus sugerencias, comentarios y apreciaciones. También agradezco a dos de los evaluadores anónimos sus apreciaciones que han ayudado notablemente a mejorar la última versión del trabajo. Este trabajo ha sido financiado parcialmente por el centrA. No obstante, cualquier error que pudiera permanecer es de mi exclusiva responsabilidad.

E-mail: pbg@ujaen.es

Revista Internacional de Sociología (RIS)

Tercera Época, n 35, Mayo-Agosto, 2003, pp. 177-197. 


\section{INTRODUCCIÓN}

El estudio del comportamiento religioso de los agentes, o de los efectos "sociales" de la religión en general, no es ni mucho menos un campo de investigación novedoso. Incluso en el seno de la economía, ya los clásicos se preocuparon por el papel de los pastores (sacerdotes) y el efecto de la religión como institución en la estructura de la economía (véase, por ejemplo, Adam Smith, [1776] 1965).

Hasta los setenta, el sector (económico) de la religión fue considerado como una actividad de "no mercado" y, por tanto, fuera del campo de estudio del análisis económico. Sin embargo, desde el trabajo de Stark (1972) y, sobre todo, a partir del ya clásico de Azzi y Ehrenberg (1975) (A-E en adelante), la Economía de la Religión aparece como una sub-disciplina de la microeconomía, utilizando las mismas herramientas analíticas que los modelos de asignación del tiempo de la economía de la familia "a là Becker".

A partir de los ochenta, se ha trabajado en esta línea de investigación de manera creciente; concretamente, tres han sido las áreas de estudio en Economía de la Religión. Por un lado, el efecto de los factores económicos en la actividad (dedicación) religiosa, _ ¿Cómo un incremento salarial modifica la asistencia a la iglesia?- Por otro, la influencia de la pertenencia a una determinada religión o congregación (a la que en términos económicos denominamos "club") en variables socio-económicas relevantes como fertilidad, salarios (ganancias), educación, movilidad laboral, sindicación, etc. Por último, trabajos relacionados con organización industrial, esto es, la función objetivo de las empresas productoras del bien religioso (las iglesias o congregaciones) y las decisiones estratégicas en los mercados.

Sobre el primer aspecto, podríamos resumir que el modelo de A-E (1975) —con las variaciones de Long y Settle (1977), Grossbard-Shechtman y Neuman (1986), Neuman (1986), Rodero y Brañas-Garza (2000) o Rodero et al. (2002) - plantea el problema del consumidor religioso como una caso normal de maximización, en el que la utilidad no sólo se deriva del consumo de bienes terrenales sino también de una commodity religiosa; en algunos casos, como en A-E, la restricción presupuestaria se ve afectada por un tiempo dedicado a la actividad religiosa (que supone un coste de oportunidad en términos de salario), mientras que en otros, se utiliza directamente un precio de mercado para los bienes religiosos (véase Rodero y Brañas-Garza (2000)).

En contra de autores como Lenski (1963), que consideran la práctica religiosa como un acto primitivo y falto de toda racionalidad, estos trabajos sobre comportamiento (económico) religioso ofrecen distintos motivos racionales que llevan a los individuos a practicar la religión: motivo salvación (aversión al riesgo), social (consumo) y profesional (comportamiento estratégico).

Sobre el segundo tipo de investigación, aquella que relaciona la pertenencia con variables socio-económicas, encontramos una gran cantidad de trabajos empíricos 
que han tratado de aproximar (cuantificar) la importancia que la pertenencia a determinadas congregaciones tiene sobre las decisiones de fertilidad, oferta de empleo, probabilidad de conflicto matrimonial, etc. (ver Neuman y Ziderman (1986) o Lehrer (1996) como ejemplos obvios). Sin embargo, aunque las aportaciones teóricas son prácticamente inexistentes, podríamos citar las investigaciones seminales de Barro y McCleary (2002) y Sacerdote y Glaeser (2001) sobre el papel de la congregación en la educación y ésta en el crecimiento económico.

Muy recientemente, aparece una tercera línea de investigación fundamentalmente teórica ${ }^{1}$, dedicada al estudio de las iglesias como empresas que producen bienes religiosos. Concretamente, Iannaccone (1992) se preocupa por la existencia de free-riders dentro de los "clubs" religiosos (congregaciones) y Stonebraker, (1993) por el tamaño óptimo de las empresas; Olds (1994) se centra en la financiación de las iglesias; Sawkins y Paterson (1996), en cómo la formación de los pastores afecta a la captación de clientes; y Rodero y Brañas-Garza (2000) "en la estructura monopolística del mercado y la fijación de precios religiosos.

Este trabajo se engloba en la primera línea de investigación desde una perspectiva empírica. Pretende analizar si las teorías que proponen los modelos de comportamiento religioso de los agentes arrojan algún tipo de evidencia para el caso español, utilizando para ello una base de datos del CIS (Centro de Investigaciones Sociológicas).

Hemos de poner de manifiesto una diferencia importante de partida: ni en España hay un nivel de competencia entre empresas religiosas comparable a los Estados Unidos (foco de la gran mayoría de trabajos, véase A-E, Erenberg (1977) y otros), ni el nivel de identificación religión-política en nuestro país es similar al caso de Israel (segundo caso más estudiado, véase Neuman (1986), Neuman y Ziderman (1986) y otros).

Partiendo de esta premisa, hemos de adelantar que nuestra base de datos (como veremos en el apartado 3) es bastante más amplia y adecuada que la utilizada en otros casos, ya sea en el tipo de preguntas que se realiza, o en el tamaño muestral utilizado.

Por otro lado, y hasta donde el autor conoce, éste es el primer estudio realizado para nuestro país desde la perspectiva "del consumo de bienes religiosos", dado que el trabajo de Brañas-Garza y Neuman (2003) se centra en exclusiva en capital humano religioso, estimando simultáneamente ecuaciones de religiosidad y educación para obtener conclusiones acerca del papel de la educación religiosa en la formación de los individuos.

\footnotetext{
' También encontramos algún trabajo empírico que trata de explicar bajo qué estrategias han actuado las distintas iglesias. Si bien los artículos de Finke y Stark (1992) (que explica la expansión americana) o Iannaccone y Stark (1994) (analizando la secularización europea) son anteriores, sin lugar a dudas, el de Ekelund et al. (1996) (que estudia el poder de monopolio de la iglesia católica en la época de la Reforma) ha sido el de mayor repercusión.
} 
RIS

REVISTA INTERNACIONAL UE SOCIOLOCIA

$\mathrm{N}^{\circ} 35$, Mayo-Agosto, 2003

PABLO BRAÑAS-GARZA

Tras esta introducción, el trabajo se divide en cinco partes. La segunda sección analiza las creencias religiosas de los españoles. La tercera presenta los modelos teóricos de comportamiento religioso. La cuarta estudia la base de datos. Los resultados del trabajo se analizan en la quinta sección. La sexta concluye y discute los resultados.

\section{CREENCIAS RELIGIOSAS EN ESPAÑA}

Comenzaremos este trabajo del mismo modo que otros dedicados a Economía de la Religión - (véase el ya clásico de Iannaccone (1998)—, esto es, estudiando si los encuestados muestran interés por la religión y por determinados aspectos relacionados ${ }^{2}$ (ver tabla 1: a).

Comparando con los de Iannaccone (1998), estos resultados son bastante sorprendentes. En su caso, todas las cuestiones planteadas tenían aproximadamente el mismo porcentaje de respuesta (se creía de la misma manera en Dios que en el cielo); sin embargo, en nuestro caso, llama la atención que el $75 \%$ de los encuestados cree en Dios absolutamente, pero no llegando ni al 25\% del total de los que creen absolutamente en los postulados básicos de la Iglesia Católica, tales como la existencia del cielo, del infierno, etc. Esta peculiaridad nos plantea dudas sobre dos cuestiones: ¿qué grado de correlación existe entre los españoles que creen en Dios y los que se confiesan católicos?; por otro lado, esta respuesta nos hace dudar de la efectividad del motivo "salvación" en la decisión de practicar la religión. Esto es, si los individuos no son temerosos de lo que vaya a ocurrir después de la vida terrenal (son neutrales al riesgo ante la presencia de una contingencia posible), hemos de acudir a otros motivos que justifiquen su práctica religiosa: los motivos sociales y profesionales.

Por otro lado, el elevado porcentaje que cree de manera inequívoca en Dios nos hace dudar de la efectividad de la hipótesis de secularización. Según esta teoría, conforme más desarrollada está una sociedad, menos religiosos se muestran los ciudadanos $^{3}$. Aunque no tengamos datos de períodos anteriores (para analizar la tendencia), si el $75 \%$ de los encuestados cree absolutamente en Dios, no parece razonable suponer que las creencias religiosas están decayendo.

\footnotetext{
${ }^{2}$ Un alto porcentaje de estos trabajos usa una base de datos norteamericana, The Gallup Pool, que recoge información sobre aspectos religiosos para EEUU, cada diez años, desde principios del siglo XX.

${ }^{3}$ La hipótesis de secularización arranca en Marx (1913:11-12) aunque se le atribuye principalmente a Weber (1930). Para una discusión reciente sobre esta hipótesis ver Chaves (1994); para un estudio del creciente interés por el culto religioso, desde un punto de vista de Teoría de Juegos, ver Raskovivh (1996).
} 
Tabla 1.

Creencias de los individuos

1:a Porcentajes (¿cree Ud. en ...?)

\begin{tabular}{lccccc}
\hline & Dios & VDM & Cielo & Infierno & Milagros \\
\hline Absolutamente & $\mathbf{7 5 , 5 6}$ & 23,91 & 22,07 & 15,96 & 17,85 \\
Más o menos & 1,65 & 24,68 & 23,55 & 17,20 & 23,47 \\
No demasiado & 8,36 & 12,06 & 13,46 & 17,18 & 15,03 \\
En absoluto & 8,48 & $\mathbf{2 7 , 0 1}$ & $\mathbf{2 9 , 9 8}$ & $\mathbf{3 8 , 1 8}$ & $\mathbf{3 3 , 4 8}$ \\
\hline
\end{tabular}

${ }^{*}$ ) El total, hasta el $100 \%$, se completa con los que NS/NC; VDM: vida después de la muerte; en negrita los valores máximos.

1:b Correlaciones (simples) entre variables

\begin{tabular}{lccccc}
\hline & Dios & VDM & Cielo & Infierno & Milagros \\
\hline Dios & 1,00 & 0,03 & 0,03 & 0,04 & 0,03 \\
VDM & & 1,00 & 0,61 & 0,58 & 0,51 \\
Cielo & & 1,00 & 0,85 & 0,63 \\
Infierno & & & 1,00 & 0,63 \\
Milagros & & & & & 1,00 \\
\hline
\end{tabular}

Antes de avanzar en el contenido del trabajo, observaremos si las respuestas antes analizadas (ver tabla 1:b) están relacionadas entre sí, calculando el nivel de correlación simple, o de Pearson, entre las cuestiones. De esta tabla salen dos ideas muy claras: por un lado, no existe relación alguna entre creer en Dios y creer en los otros aspectos de la vida supra-terrenal: vida después de la muerte (VDM), cielo, infierno o los milagros; por otro, sí existe una elevada correlación dentro de este segundo conjunto de creencias y, además, el binomio cielo/infierno es prácticamente inseparable.

Por tanto, parece que los individuos muestran claramente su creencia hacia un ser superior, pero no queda claro que esta creencia sea próxima a la visión de la Iglesia Católica. 
RIS

REVISTA INTERNACIONAL DE SOCIOLOGIA

№ 35, Mayo-Agosto, 2003

PABLO BRAÑAS-GARZA

\section{MODELOS TEÓRICOS DE COMPORTAMIENTO RELIGIOSO}

Los modelos teóricos que analizan el comportamiento religioso de los individuos son principalmente dos. El pionero de A-E que busca explicar los determinantes de la asistencia a la iglesia o a otras actividades relacionadas ${ }^{4}$; y el posterior de Neuman (1986), que explica el efecto de la pertenencia en la toma de decisiones familiares. Tanto los trabajos posteriores de Stark et al. (1996), como las de Rodero et al. (2002), no dejan de ser extensiones o generalizaciones de los anteriores que no cambian sustancialmente el planteamiento del modelo. Para una ilustración de este tipo de análisis formal, estudiaremos a lo largo de este apartado, un modelo de comportamiento religioso como el de A-E.

Tomamos como ejemplo a una familia compuesta por dos individuos $(j=1,2$; marido y mujer) que practican la religión, ya sea por motivo social ${ }^{5}$ (las iglesias pueden ser un lugar adecuado para establecer relaciones sociales o de vecindad), o profesional (se busca la entrada en el "club" religioso para aumentar las oportunidades profesionales ${ }^{6}$ ) o con el fin de alcanzar la salvación, ante la incertidumbre acerca de lo que hay después de la muerte. Sea cual sea el motivo que les lleva a ser religiosos, suponemos que esta práctica afecta a su nivel de utilidad. Su función de utilidad será cuasicóncava y tendrá dos argumentos: el consumo terrenal y el consumo celestial esperado ${ }^{7}$.

$$
U=U\left(C_{t}, O\right)
$$

donde $C_{t}$ es el nivel de consumo en el periodo $t$ y $O$ el valor esperado de la porción de vida después de la muerte. Para simplificar, consideraremos que la fecha de la muerte es conocida (exógena e idéntica para los dos); también será conocido (y dado) el nivel salarial presente y futuro.

La familia transforma el bien compuesto adquirido en el mercado $(x)$ a través de su esfuerzo personal (dedicándole parte de su tiempo tanto el marido $\left(h_{l, t}\right)$ como la esposa $\left(h_{2, t}\right)$ ), obteniendo así el bien de consumo deseado $\left(C_{t}\right)$. Esta función de producción será idéntica en todos los periodos y la supondremos cóncava y continua (ver ecuación [2]).

\footnotetext{
${ }^{4} \mathrm{Si}$ bien su modelo se centra exclusivamente en la asistencia (a la iglesia), resulta, como los autores proponen, fácilmente generalizable a otras actividades como donaciones, rezo, etc.

${ }^{5}$ A este motivo algunos autores, como Ulbrich y Wallace (1983), lo denominan "consumo": los individuos derivan utilidad presente de la asistencia a los actos religiosos, como una actividad de ocio más, sin ninguna relación con el motivo salvación (ver también Wallis (1990)).

${ }^{6}$ Este tipo de argumento se expone como una razón fundamental para el caso de los judíos, especialmente de los norteamericanos (ver Iannaccone (1998)).

${ }^{7}$ Porción de cielo que él espera tener en un futuro, como resultado de su esfuerzo religioso efectuado durante su vida. Como se observa no suponemos una solución binaria (cielo/infierno), sino un continuo de posibles resultados: la porción de cielo será función de la inversión terrenal.
} 
La porción esperada de cielo $(O)$ la supondremos como una función cóncava ${ }^{8}$ y continua del tiempo dedicado por la familia a actividades religiosas a lo largo de su vida. Si bien suponemos que el premio celestial tendrá duración infinita a partir del periodo $n+l$, si el individuo tiene tasa de descuento positiva, podemos transformar este flujo infinito en el valor actual descontado presente del mismo, al que llamamos $O$.

$$
\begin{aligned}
C_{t} & =C\left(x_{t}, h_{j, t}\right) \\
O & =Q\left(r_{j, t}\right) \quad \forall t
\end{aligned}
$$

siendo $r_{j, t}$ el tiempo dedicado a las prácticas religiosas por cada miembro de la familia $(j=1,2)$ en cada momento $(t=1,2, \ldots, n)$. Nótese que hay sustitución perfecta entre inputs productivos, esto es, resulta irrelevante quién sea el que dedique más tiempo a la religión ya que el beneficio esperado es compartido por ambos; por otro lado, todas las actividades preceptivas como la asistencia, la educación religiosa de los hijos, la donación, el rezo doméstico, etc. quedan incluidas en este argumento?.

Si $p$ es el precio del bien compuesto (fijo), $w_{l, t}$ el salario laboral en cada periodo para el marido y $w_{2, t}$ para la esposa, $i$ el tipo de interés constante, $v$ el nivel de riqueza no laboral de la familia y $l_{j t}$ la cantidad de horas de trabajo en cada periodo $t$, su restricción presupuestaria (descontada) a lo largo de todo su ciclo vital sería:

$$
\sum_{i=1}^{n}\left[\frac{p x_{t}}{(1+i)^{t-1}}\right]=\sum_{i=1}^{n}\left[\frac{v+w_{1, l} l_{1, t}+w_{2, l} l_{2, t}}{(1+i)^{t-1}}\right]
$$

Si $T$ representa la totalidad de tiempo del que dispone en cada período, su restricción temporal tomaría la forma:

donde $h_{j, r}, r_{j, t}, 1_{j, t} \geq 0$.

$$
\mathrm{T}=h_{\mathrm{j}, \mathrm{t}}+r_{\mathrm{j}, \mathrm{t}}+\mathrm{l}_{\mathrm{j}, \mathrm{t}} \quad \forall t ; j=1,2
$$

\footnotetext{
${ }^{8} \mathrm{La}$ concavidad se impone para evitar soluciones esquina; dicho comportamiento religioso prácticamente monástico no podría representar en ningún caso a la mayoría de la población.

${ }^{9}$ Es precisamente en estas cuestiones donde profundizan los trabajos de Neuman (1986) y Rodero et al. (2002). Por ejemplo, una de las posibilidades exploradas por este último es el intercambio de donaciones por asistencia.
} 
RIS

REVISTA INTERNACIONAL DE SOCIOLOGIA

Las ecuaciones [1-4] plantean el problema de optimización. Si las funciones de producción [2] se introducen en la [1] y si, por otro lado, despejamos el tiempo de trabajo, $l_{j, t}$, de la restricción temporal [4] y lo introducimos en la restricción presupuestaria [3], podemos obtener una restricción "completa" para todo el ciclo vital. Todo ello nos permite escribir el Lagrangiano para este problema ${ }^{10}$. Asumiendo que hay solución interior, las condiciones de primer orden (CPO) requieren que en el óptimo:

$$
\frac{\partial Q / \partial r_{1, t}}{\partial Q / \partial r_{2, t}}=\frac{w_{1, t}}{w_{2, t}}
$$

Es decir, la dedicación de cada miembro familiar a la producción del bien religioso será una proporción de sus salarios laborales; si el salario del marido es mayor que el de la mujer, entonces, la esposa tendrá que dedicar más tiempo a actividades religiosas.

Si suponemos que el marido y la mujer mantienen sus salarios constantes a lo largo del tiempo, de las CPO también se deriva que:

$$
\frac{\partial Q / \partial r_{1, t}}{\partial Q / \partial r_{2, t}}=(1+i)^{-1} \quad \forall t, j=1,2
$$

Sin embargo, si los salarios varían en el tiempo, esto es, si entre dos períodos $t$ y $t-l$ observamos un cambio de los salarios, entonces, la condición [6] se convierte en,

$$
\frac{\partial Q / \partial r_{1, t}}{\partial Q / \partial r_{2, t}}=\frac{w_{j, t}}{w_{j, t-1}}(1+i)^{-1} \quad \forall t, j=1,2
$$

De lo cual deducimos que, si el patrón (a lo largo del ciclo vital) de ingresos salariales tiene forma de " $U$ " invertida, entonces la tendencia temporal de la práctica religiosa ha de mostrar forma de "U".

$$
\begin{aligned}
{ }^{10} \text { El Langrangiano sería: } \mathrm{L}= & U\left[C\left(x_{1} h_{1,1}, h_{2,1}\right), C\left(x_{2} h_{1,2}, h_{2,2}\right), \ldots C\left(x_{n} h_{1, n}, h_{2, n}\right), Q\left(r_{1}, \ldots r_{n}\right)+\right. \\
\lambda & \lambda\left(\sum_{i=1}^{n}\left[\frac{p x_{t}}{(1+i)^{t-1}}\right]-\sum_{i=1}^{n}\left[\frac{\mathrm{v}+\sum_{j=1}^{2} \mathrm{w}_{j, t}\left(T-h_{j, t}-\mathrm{r}_{\mathrm{j}, t}\right)}{(1+i)^{t-1}}\right]\right)
\end{aligned}
$$


De manera resumida, este modelo parece ser capaz de explicar por qué las mujeres van más a la iglesia que los hombres (o por qué los habitantes de pueblos pequeños también acuden con mayor frecuencia que los de grandes ciudades), puesto que los salarios son menores para los primeros que para los segundos; del mismo modo, explica la asistencia más acusada de las personas conforme aumenta su edad aunque teniendo en cuenta cómo mejoran (empeoran) sus ingresos laborales. En los dos primeros puntos analizaremos empíricamente estas cuestiones.

La versión de Rodero et al. (2002) introduce la sustitución perfecta entre todos los inputs productivos dedicados a la producción de output religioso; esto es, los individuos eligen entre las distintas opciones de práctica religiosa que se les ofrece para realizar su inversión (asistencia a la iglesia, rezo doméstico, etc.). En el tercer apartado del capítulo de resultados, analizaremos si los individuos sustituyen, realmente, esos factores productivos.

Neuman (1986) hace una versión estática del modelo A-E, en el que un individuo aislado puede comprar bienes de consumo y bienes religiosos. Estos bienes tienen un precio de mercado (exógeno y fijo) y el individuo dedica un tiempo en el consumo de cada uno de ellos; esa cantidad de tiempo empleada en la religión es el foco del modelo de Neuman y de su aplicación empírica al caso de Israel (definiendo para ello ecuaciones de asistencia). De manera resumida, su modelo indica que el tiempo dedicado a la asistencia (church-attendance) está relacionado positivamente con el tiempo total disponible del individuo y el precio de los bienes de consumo; $y$, negativamente, con el nivel de riqueza familiar (no laboral), salarios y horas de trabajo. Por último, que la relación entre la asistencia y el precio del bien religioso y el tiempo dedicado al consumo de bienes terrenales es ambigua. En el cuarto subapartado del capítulo de resultados repetiremos la batería de estimaciones de la ecuación de asistencia de Neuman para el caso de nuestro país.

En el último apartado de resultados indagaremos en la relación existente entre la práctica religiosa de los individuos y el ejemplo de los padres. Trabajos recientes, como los de Grossbard-Shechtman (1999) o Tamura (1999), analizan el papel de la familia en la decisión individual de adherirse a una religión y practicarla.

\section{LA BASE DE DATOS}

Los datos utilizados en este trabajo provienen de la encuesta realizada por el Centro de Investigaciones Sociológicas (CIS) dentro del programa International Social Survey Program (ISSP): Religion, financiado por la UNESCO (código del CIS: estudio 2301). Dicha encuesta fue realizada en 1998 por medio de entrevistas personales en el ámbito nacional, para un universo de población de españoles mayores de edad en 147 poblaciones correspondientes a 47 provincias; se proyec- 
taron 2.488 entrevistas y se realizaron 2.488. El nivel de confianza es del $95,5 \%$, con un error del $2 \%$, suponiendo que se trata de un muestreo aleatorio simple. A continuación se presentan las principales ventajas y limitaciones derivadas del uso de esta base de datos.

Ventajas. Con respecto a las utilizadas en los trabajos de A-E y Neuman (1986) la mejora es notoria. El tamaño muestral de A-E era de 49 individuos (Estados americanos), mientras que el de Neuman no llegaba a 200. En el primero se utilizaba la media de los valores en cada Estado de los EEUU ${ }^{11}$; en el segundo, se usaba un estudio piloto. Por tanto, esta encuesta no sólo aumenta significativamente el tamaño de la muestra, sino que también tiene ámbito nacional, lo cual permite generalizar, en algún sentido, los resultados. Por último, los datos provienen de un estudio que ha sido explícitamente diseñado para ello, lo que no era el caso de los anteriores.

Limitaciones. Los datos aportados presentan dos limitaciones importantes: por un lado, se han categorizado casi todas las variables incluso aquéllas de naturaleza continua (por ejemplo, niveles salariales); por otro, no hay información alguna ni sobre las donaciones a las iglesias (church-giving), ni sobre el tiempo dedicado a la educación (religiosa) de los hijos ${ }^{12}$; ambas cuestiones limitan la extensión del estudio.

\section{RESULTADOS}

\section{Asistencia a la Iglesia de hombres y mujeres: evidencia empírica}

Como se observó en el apartado de creencias religiosas en España, los modelos teóricos infieren el reparto de las actividades religiosas entre los miembros de la familias. De la ecuación [5] se deduce que los maridos acuden con menor frecuencia a la iglesia que las esposas ya que su nivel salarial es mayor y, por tanto, para la familia resulta más beneficioso - en términos de coste de oportunidad-que sea la esposa la que realice esas actividades.

Las siguientes dos figuras presentan las funciones de densidad de la asistencia (church-attendance) tanto de los hombres como de las mujeres ${ }^{13}$. Dado que los

\footnotetext{
${ }^{11}$ Además, en muchos casos, las observaciones para los distintos Estados no venían de la misma fuente.

${ }^{12}$ Aunque, curiosamente, sí disponemos del tiempo (medido a través del ejemplo) que los padres dedicaron para adherir a sus hijos a la práctica religiosa.

${ }^{13}$ Hemos de destacar que el $64 \%$ de las mujeres y el $67 \%$ de los hombres de la muestra están casados, de los cuales el $97 \%$ y $94 \%$, respectivamente, declaran que su esposo/a es católico/a. En este contexto, las hipótesis que plantean que las diferencias religiosas entre cónyuges aumentan las probabilidades de divorcio (ver Lehrer, 1996) no resultarían relevantes para el caso español.
} 
datos son de naturaleza discreta se utiliza un kernel que aproxima dichas funciones de densidad.

Ambas figuras parecen confirmar lo expuesto anteriormente. En la medida que, en nuestro país, los salarios de los hombres son mayores que los de las mujeres, son éstas las que fabrican mayoritariamente el output religioso, medido en esta ilustración a través de la asistencia. Las mujeres encuestadas (casadas y solteras) se concentran en niveles de asistencia elevados, mientras que, en el caso de los hombres dicha asistencia es mucho menos regular.

Figura 1.

Asistencia a la iglesia (por sexo)

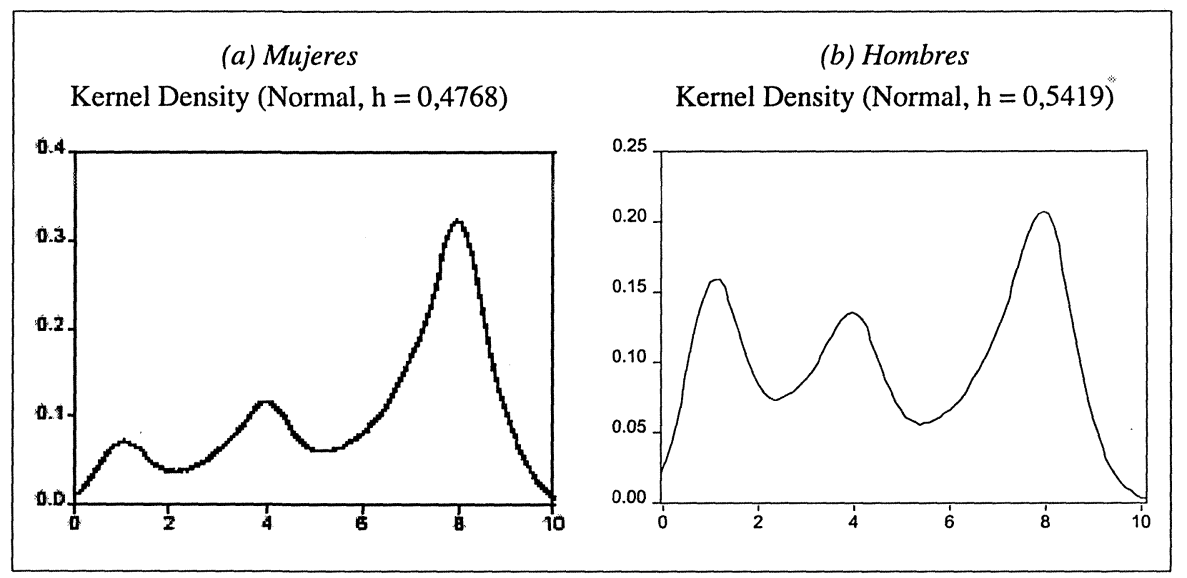

Por otro lado, estos resultados coinciden con los planteados por la Psicología de la Religión, aunque, a diferencia de ésta, que considera que la mayor religiosidad de las mujeres frente a los hombres se debe a razones de género, para los economistas son los costes de oportunidad los que generan estas diferencias (ver Beit Hallahmi, 1997). Por ello, en el caso de nuestro país, donde un porcentaje importante de mujeres (esposas) no trabaja (o lo hace a tiempo parcial), este resultado tiene mucho más sentido, dado que los costes de oportunidad del tiempo del hombre (marido) serán mucho mayores ${ }^{14}$.

En Brañas-Garza y Neuman (2003) se analiza la composición de las rentas laborales de los matrimonios y se constata que, en los casos donde la aportación de las esposas es menor, mayor es su actividad religiosa (medida, tanto en asistencia, como en rezo doméstico).

${ }^{14}$ Estamos suponiendo una sustitución perfecta entre inputs productivos, es decir, es indiferente que sea el marido o la mujer el que aporte su tiempo. 


\section{La relación entre la asistencia a la Iglesia y la edad}

Otra de las cuestiones que derivamos de los modelos teóricos es que existe una relación inversa entre el patrón temporal de ingresos (ganancias salariales) y nivel de asistencia en el tiempo. Dado que este patrón de ingresos tiene de manera característica la forma de "U invertida" (tipo $\mathrm{ABM}$ ) ${ }^{15}$, esperamos ver la relación en forma de $U$ para el caso de la asistencia a la iglesia.

La figura 2.a ilustra la asistencia media de los individuos ${ }^{16} \mathrm{de}$ la misma edad. Ordenando dichos valores de modo creciente (en la edad) tratamos de extraer el patrón temporal de dicha actividad para el conjunto de los ciudadanos.

Figura 2.

Relación entre asistencia y edad

(a) valores medios ordenados

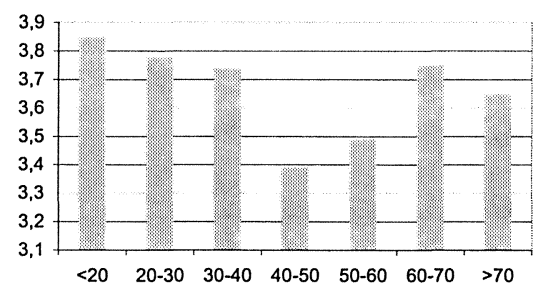

(b) Valores muestrales y filtro

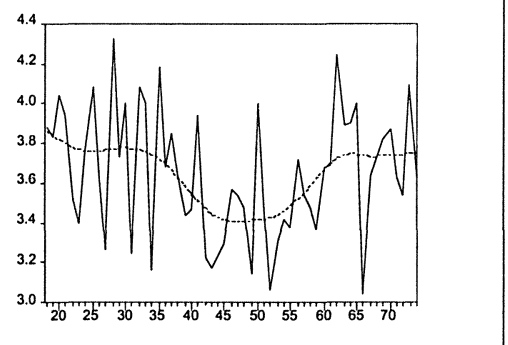

(*) Eje de las X: individuos ordenados por edad; eje de las Y: asistencia media a la iglesia. (**) La línea discontinua es un filtrado (de Hodrick-Prescott) de los datos muestrales.

Los datos parecen evidenciar lo que la ecuación [7] nos planteaba. Existe una relación inversa entre el patrón de salarios y el de asistencia a la iglesia. A través del filtrado de los datos (ver figura 2.b) se observa con bastante nitidez la forma de " $U$ ". La parte plana de los jóvenes (hasta los 30 años aproximadamente) nos muestra un tramo en el cual la asistencia permanece constante. Dos argumentos

\footnotetext{
${ }^{15}$ Ando-Burmberg-Modigliani suponen que los ingresos derivados del trabajo son reducidos cuando el individuo entra en el mercado laboral y que aumentan conforme alcanza su madurez profesional; posteriormente, caen conforme se acerca la edad del retiro (ver en cualquier manual de macroeconomía intermedia).

${ }^{16}$ Todos los encuestados: hombres y mujeres, casados y solteros.
} 
pueden justificar este hecho: por un lado, los salarios podrían ser lo suficientemente bajos en las primeras etapas de la vidad profesional; por otro, tendríamos un porcentaje de jóvenes todavía no incorporados al mercado laboral (y, por tanto, el coste de oportunidad del tiempo dedicado a actividades religiosas sería cero en términos salariales).

A edades avanzadas también encontramos un tramo plano, donde la edad no afecta a la decisión sobre la asistencia. Si bien parece que tendría que aumentar la asistencia, ya que la contingencia posible está más próxima; encontramos dos argumentos que justifican la constancia en la asistencia. Por un lado, los sujetos podrían considerar (bajo un esquema de planificación intertemporal) que ya han realizado suficiente inversión en capital religioso (ver Neuman, 1986) y deciden no incrementar su inversión neta. Por otro, los individuos podrían tener enormes costes de transporte (por problemas de salud) que dificultarían su asistencia a la iglesia.

Nuevamente, parece que las predicciones del modelo de A-E se adaptan razonablemente bien al comportamiento de los españoles.

\section{Sustituibilidad entre distintas actividades religiosas}

En algunos trabajos recientes, como en Sullivan (1985), Tamura (1999) o Rodero et al. (2002), se plantea la idea de que los individuos pueden sustituir distintas actividades religiosas según su interés. En la línea de la Economía de la Familia, los agentes pueden preferir invertir más tiempo doméstico en la producción de bienes y acudir con menor frecuencia al mercado (de bienes de consumo y religiosos). En este sentido, se argumenta que, debido a los costes de transporte y a la rigidez de los horarios laborales, la asistencia a la iglesia puede tener costes crecientes, provocando que los individuos sustituyan el bien ofrecido en el mercado (el servicio religioso) por otro de producción "casera": el rezo.

Este planteamiento nos llevaría a encontrar niveles de correlación negativos y significativos entre asistencia (a los servicios religiosos $\mathrm{u}$ otras actividades de la congregación) y rezo doméstico. La tabla 3 analiza esta cuestión (el cuadrante resaltado dentro de la tabla muestra las correlaciones entre estas tres variables).

Las correlaciones no nos llevan a extraer ninguna conclusión en este sentido. Si bien la relación rezo-asistencia (iglesia) es negativa, su valor es tan reducido que no aporta prácticamente nada. En los otros casos: rezo-asistencia (otras actividades religiosas) parece tener cierta relación positiva, mientras que para asistencia (iglesia)-asistencia (otras) volvemos a una relación casi nula.

Por tanto, para el caso de los españoles, no parece existir relación de sustitución alguna entre los inputs religiosos, sino que, más bien, éstos son factores independientes. Únicamente se podría observar alguna relación de complementariedad (débil) entre rezo doméstico y asistencia a otras actividades de la congregación. 


\section{Determinantes de la asistencia a la Iglesia}

Como vimos al final del segundo apartado, la extensión de Neuman del modelo de A-E permitía obtener una ecuación de asistencia a la iglesia. Para comparar sus resultados en Israel con los nuestros y extraer alguna información sobre los determinantes de la asistencia, repetiremos ${ }^{17}$ la misma batería de estimaciones para el caso español (ver tabla 2).

Si bien los resultados - por los problemas estadísticos comentados anteriormente - son bastante pobres, los signos esperados de los parámetros estimados $(\hat{\beta})$ parecen ajustarse bien a los ofrecidos en el trabajo de Neuman, a excepción de la "formación", que en su caso resultaba negativo.

Tabla 2.

Estimación de las ecuaciones de asistencia a la Iglesia

\begin{tabular}{lcccc}
\hline \multicolumn{5}{c}{ Asistencia a la iglesia: $y=f\left(x_{1}, \ldots, x_{n}\right)$} \\
Var. Exóg. $(x)$ & Regr. 1 & Regr. 2 & Regr. 3 & Regr. 4 \\
\hline Edad & $0,09(9,28)$ & $0,10(10,36)$ & $0,04(0,87)$ & $0,04(1,81)$ \\
Edad $^{2}$ & -- & -- & $0,00(0,93)$ & $-0,00(-0,10)$ \\
Ingresos & $0,07(0,75)$ & --- & $0,08(0,81)$ & -- \\
Formación & -- & $0,06(2,28)$ & -- &.-- \\
Número Hijos & $0,31(2,85)$ & $0,22(2,26)$ & $0,31(2,89)$ & $0,13(2,65)$ \\
Tradición materna & $0,76(0,51)$ & $0,68(0,50)$ & $0,82(0,56)$ & $-0,97(-1,51)$ \\
Ejemplo materno & $0,38(4,66)$ & $0,45(6,13)$ & $0,38(4,63)$ & $0,18(4,66)$ \\
Tradición paterna & $-0,68(-0,85)$ & $-1,26(-1,65)$ & $-0,72(-0,90)$ & $-0,73(-1,90)$ \\
Ejemplo paterno & $0,31(4,27)$ & $0,22(3,30)$ & $0,31(4,30)$ & $0,21(6,17)$ \\
Tradición esposa & $0,00(0,00)$ & $-0,12(0,43)$ & $0,13(0,36)$ & $-0,22(-1,32)$ \\
C & $1,88(1,18)$ & $1,80(1,25)$ & $2,77(1,49)$ & $2,88(3,66)$ \\
\hline $\mathrm{R}^{2}$ ajustado & 0,17 & 0,18 & 0,17 & 0,14 \\
\hline
\end{tabular}

$\left(^{*}\right)$ entre paréntesis la $t$-student.

De los coeficientes estimados (ver tabla 2) que han resultado estadísticamente significativos es interesante destacar lo siguiente:

\footnotetext{
${ }^{17}$ Dados los niveles de multicolinealidad que se aprecian, la estrategia razonable sería estimar por componentes principales y en logaritmos (para medir las elasticidades). Sin embargo, dada la naturaleza de nuestros datos, nos limitaremos a repetir la estimación de Neuman.
} 
(1) La edad afecta (positivamente) a la asistencia cuando la renta no está presente; obviamente, por la idea del ciclo vital, estas variables están muy relacionadas.

(2) El tamaño de la familia también se relaciona positivamente con la asistencia; por tanto, los costes de transporte (que disminuyen la asistencia) no compensan los beneficios «sociales» que la familia obtiene de la mayor asistencia a la congregación.

(3) El ejemplo de la madre (padre) aumenta la asistencia; si bien la tradición familiar es irrelevante, el ejemplo de los progenitores resulta positivo ${ }^{18}$. Por tanto, la inversión de los padres es un determinante positivo de la asistencia.

(4) La educación (regresión 2) es significativa y tiene un efecto positivo sobre la asistencia, esto es, a mayor nivel educativo, mayor frecuencia de asistencia al club.

Resumiendo, la edad, la inversión en capital humano religioso, el número de hijos y el nivel educativo parecen ser los determinantes (positivos) de la asistencia. Lo primero se deriva de los modelos teóricos, lo segundo, lo veremos en el siguiente apartado, pero, ¿por qué el tamaño de la familia y la educación se relacionan positivamente con la asistencia?

Para contestar a esta pregunta recurriremos al motivo profesional (comportamiento estratégico). En principio, parece obvio que los individuos de nivel educativo más alto son los que más rendimientos salariales obtienen, por lo que, deberían ser los que menos acuden a la iglesia (el signo debería ser negativo); por lo tanto, hemos de encontrar otra razón que les compense ese gasto de tiempo. Si los individuos con mayor formación asisten con mayor regularidad, los individuos que estratégicamente persigan estas relaciones (professional linkages) tendrán que acudir a estas congregaciones; además, esta estrategia sólo tendrá éxito si el free-rider (el que se pretende aprovechar de los clubs religiosos) tiene un nivel mínimo de educación. Dicho de otro modo: sólo las personas con mayor educación podrán obtener rendimientos de dichas redes y el beneficio de éstas será mayor que el coste en términos de tiempo ${ }^{19}$.

Para Barro y McCleary (2002) la correlación entre religión y educación se debe a que la práctica religiosa requiere de un pensamiento abstracto y complejo; por tanto, se requiere cierto nivel intelectual para poder asimilar dichos planteamientos. Entonces, tiene sentido una correlación positiva entre actividad religiosa y educación.

\footnotetext{
${ }^{18}$ En la medida en que el "ejemplo" es una actividad que requiere tiempo, y, por tanto, tiene un coste de oportunidad positivo, se podría asimilar esa variable a la inversión en capital religioso. Dicha inversión no sería one-shot (una sola vez) sino continua, esto es, un plan de inversión distribuido en el tiempo.

${ }^{19}$ Aducir al motivo "salvación" no tendría sentido alguno ya que podrían comprar otras commodities religiosas, como el rezo doméstico, donde ni incurren en costes de transporte, ni obtienen beneficios de la red (comunidad religiosa o congregación).
} 


\section{RIS}

Sobre el tamaño de la familia surge la misma cuestión: Conforme mayor resulta el tamaño de la misma, mayor es el coste que supone el transporte. Teniendo como alternativa el rezo doméstico, ¿por qué acuden a la congregación? Solamente el motivo profesional o el social pueden explicar esta conducta; esto es, los beneficios esperados (sociales y/o profesionales) han de ser mayores que los costes de asistencia y ambos han de crecer con el tamaño de la familia.

\section{La idea del ejemplo en la formación del capital humano}

Tanto Grossbard (1999) como Tamura (1999) profundizan en el papel de la familia en la educación religiosa de los individuos (la inversión en capital religioso). De alguna manera, necesitamos conectar a los individuos con la religión (al menos la primera vez) y, según estos autores, es la familia la que vincula a los agentes a estas prácticas.

Como se comentaba anteriormente, los padres hacen una inversión continua (el ejemplo) con la intención de que sus hijos se adhieran (introduzcan) a un determinado club religioso. Si bien las razones que llevan a la familia a educar a sus hijos (religiosamente) podrían ser las mismas que las anteriormente expuestas para el motivo social y profesional, en el motivo salvación encontramos algún matiz relevante. Si la religión no estipula claramente los premios (rendimientos) que la adhesión de un nuevo miembro tiene para el que lo introduce, el individuo no tiene incentivos para buscar nuevos miembros. Por tanto, estaríamos ante un caso de altruismo (puro) de los padres hacia los hijos.

Gracias a la variedad de esta base de datos podemos arrojar alguna evidencia suplementaria a otros trabajos existentes ya que, no sólo conocemos la tradición religiosa del padre o de la madre, sino también su nivel de práctica según fue percibido por el hijo, esto es, el ejemplo.

Las estimaciones recientemente estudiadas (tabla 2) aportaban cierta relevancia a esta conducta ejemplar, aunque sólo para el caso de la asistencia. Utilizaremos en este apartado (ver tabla 3) la correlación simple entre el nivel de asistencia a la iglesia, la asistencia a otras actividades, el rezo (los tres se refieren a la práctica actual del individuo) y la conducta (religiosa) del propio individuo cuando era adolescente, con las variables familiares observadas relativas a la inversión en capital religioso: el ejemplo del padre y el de la madre.

De estas correlaciones (simples) que eliminan los problemas de multicolinealidad de las estimaciones anteriores, extraemos un conjunto de ideas interesantes que ponderan los resultados anteriores:

(1) Los ejemplos del padre y de la madre no tienen relación alguna entre sí, y si la relación existe ésta es negativa, lo que podría indicar cierta división de tareas dentro de la familia. 
Tabla 3.

Actividad religiosa y ejemplo familiar

\begin{tabular}{lcccccc}
\hline & Ej. Padre & Ej. madre & Rezo & Asist. (ig.) & Asist. (ot.) & Adolesc. \\
\hline Ej. Padre & 1,00 & $-0,02$ & 0,27 & $-0,00$ & 0,02 & 0,45 \\
Ej. Madre & & 1,00 & 0,02 & $-0,06$ & 0,34 & 0,00 \\
Rezo & & & 1,00 & $-0,03$ & 0,12 & 0,39 \\
Asist. (ig.) & & & & 1,00 & 0,03 & $-0,04$ \\
Asist. (ot.) & & & & & 1,00 & 0,05 \\
Adolesc. & & & & & & 1,00 \\
\hline
\end{tabular}

(2) El efecto de este ejemplo sobre el nivel de asistencia actual a la iglesia es, en el mejor de los casos, irrelevante cuando no negativo.

(3) Sin embargo, el ejemplo del padre resulta muy relevante en lo que respecta a la asistencia durante la adolescencia y al nivel actual de rezo.

(4) El ejemplo de la madre (y no del padre ${ }^{20}$ ) afecta positivamente a la asistencia actual a las actividades organizadas por la congregación.

En resumen, parece que el papel de los padres en la dedicación religiosa actual es variado y podría ser una manifestación de la división de tareas (especialización) que caracteriza los modelos de asignación del tiempo de la familia a là Becker. Por un lado, el padre parece tener un papel importante durante la adolescencia y en el nivel actual de rezo; la madre, por el contrario, parece influir (o tener mayor peso) en la asistencia a las actividades organizadas por la congregación. Por último, y matizando los resultados presentados anteriormente, el nivel actual de asistencia a la iglesia no parece estar relacionado con la conducta de los padres.

\section{CONCLUSIONES}

Este trabajo ha tratado de introducir el estudio del comportamiento religioso de los agentes desde la perspectiva del análisis económico de la religión para el caso español. A pesar de su carácter introductorio y general, que no pretende entrar con detenimiento en ninguno de los campos específicos de la materia, permite intuir una serie de cuestiones que pueden servir como una primera aproximación al objeto de estudio. Concretamente, consideramos que los resultados más interesantes del trabajo son los siguientes:

\footnotetext{
${ }^{20}$ Lo cual parece razonable si son las madres las que practican estas actividades, de acuerdo con lo analizado en anteriores apartados. Este argumento refuerza la idea de la división de tareas (reparto y especialización) de las actividades domésticas.
} 
Resultado 1: Los postulados básicos del modelo de A-E parecen encontrar un buen acomodo en los datos españoles. Por un lado, una mayor asistencia de las mujeres que de los hombres; por otro, una trayectoria temporal en la asistencia inversa a la de los ingresos salariales.

Resultado 2: La edad, el número de hijos y la formación parecen ser los determinantes (positivos) de la asistencia. Aunque los niveles de significación de las distintas estimaciones resulte pobre, dichas variable son significativamente distintas de cero y positivas, lo que indica que incrementan la asistencia.

Resultado 3: El ejemplo de los padres no parece determinante en la asistencia a la iglesia y observamos una clara división de tareas: el ejemplo del padre es determinante en el rezo (actual) y en la práctica durante la adolescencia; la madre, por el contrario, influye positivamente en la asistencia a las actividades de la congregación.

Todo esto nos lleva a replantearnos si es el motivo salvación el determinante de la práctica religiosa, o si los motivos sociales y profesionales tienen mayor peso. Los resultados de las estimaciones de los dos últimos subapartados del capítulo de resultados nos llevan a pensar que todos ellos están presentes en la actividad religiosa. Tanto la aversión al riesgo, como los motivos terrenales, encuentran un acomodo razonable para el caso de los españoles.

Sin embargo, queda un enorme camino por explorar. El trabajo de BrañasGarza y Neuman (2003) estudia con mucha profundidad el papel de la educación en la actividad religiosa y concluye que los motivos profesionales parecen mucho más relevantes para los individuos cualificados. Pero quedan muchas cuestiones pendientes que requieren de una investigación mucho más exhaustiva.

Por un lado, el estudio del sector productivo, esto es, el análisis de la producción de bienes religiosos por parte de las empresas. En el caso de nuestro país, podríamos hablar prácticamente de un monopolio, como proponían Ekelund et al. (1996) para el medievo. La teoría de la competencia imperfecta nos dice que la mejor estrategia del monopolista sería la diferenciación del producto, por tanto, la pregunta a responder sería si la Iglesia Católica practica dicha diferenciación.

Por otro lado, queda sin resolver el papel de las donaciones. En la medida que éstas pueden sustituir a otras prácticas, como plantea Sullivan (1985), sería interesante conocer cómo se relacionan donaciones, renta y asistencia en nuestro país. Probablemente, los resultados no sean tan obvios como se intuyen.

Por último, sería interesante realizar una comparación temporal de resultados, cuando nuevas versiones de la base de datos (con nuevas observaciones) estén disponibles. Muy probablemente, cuando las cohortes que participen en la encuesta varíen, los resultados serán distintos, lo que indicará un cambio en ciertos patrones de comportamiento. 


\section{REFERENCIAS BIBLIOGRÁFICAS}

AZZI, C. y R.G. EHRENBERG (1975), "Household Allocation of Time and Church Attendance", Journal of Political Economy, $\mathrm{n}^{\circ} 83:$ (1), pp. 27-56.

BARRO, R. J. y M.M. RACHEL (2002), "Religion and Political Economy in an International Panel," NBER, Working Paper, nº 8931.

BEIT-HALLAHMI, B. (1997), "Biology, Density and Change: Women's Religiosity and Economic Development", Journal of Institutional and Theoretical Economics, $\mathrm{n}^{\circ} 153$ (1), pp. 166-178.

BRAÑAS-GARZA, P. y N. NEUMAN (2004), "Religiosity within an Economic Framework: the Case of the Spanish Catholics", Review of Economics of the Household, en prensa 2004.

CHAVES, M. (1994), “Secularization as Declining Religiosity Authority”, Social Forces, n⿳ 72(3), March, pp. 749-774.

EHRENBERG, R.G. (1977), "Household Allocation of Time and Religiosity: Replication and Extension", Journal of Political Economy, n 85 : 2, pp. 415-23

EKELUND, R. B., R.F. HEBERT y R.D. TOLLISON (1996), Sacred Trust: The Medieval Church as an Economic Firm, New York, Oxford U. Press.

FINKE, R. y R. STARK (1992), The Churching of America 1776-1990, New Brunswick Nj, Rutgers University Press.

GROSSBARD-SHECHTMAN, S. A. (1999), "Models of Marriage Markets", Economy and Society, pp. 105-126, Cambridge Univ. Press.

GROSSBARD-SHECHTMAN, S. A. y N. NEUMAN (1996), "Economic Behavior, Marriage and Religiosity”, Journal of Behavioral Economics, n 15, pp. 71-86.

IANNACCONE, L.R. (1992), "Sacrifice and Stigma: Reducing Free-Riding in Cults, Communes, and Other Collectives", Journal of Political Economy, $\mathrm{n}^{\circ}$ 100: 2, pp. 271-97.

(1998), "Introduction to the Economics of Religion", Journal of Economic Literature, $\mathrm{n}^{\circ} 36$, 1465-1496.

IANNACCONE, L.R. y R. STARK (1994), “A Supply-Side Reinterpretation of the 'Secularization' of Europe", Journal of the Scientific Study of Religion, n³3, March, 76-88.

LENSKI, G.E. (1963), The Religious Factor, Garden City, NY, Doubleday.

LEHRER, E.L. (1996), "Religion as a Determinant of Marital Fertility", Journal of Population Economics, nº 9, pp. 173-196.

LONG, S.H. y F.S. RUSSELL (1977), "Household Allocation of Time and Church Attendance: Some Additional Evidence", Journal of Political Economy, n 85: 2, pp. 409-13. 
RIS

REVISTA INTERNACIONAL DE SOCIOLOGIA

No 35, Mayo-Agosto, 2003

PABLO BRAÑAS-GARZA

MARX, K. (1913), A Contribution to the Critique on Political Economy [Traducción inglesa de N.I. Stone], Chicago, Kerr \& Co.

NEUMAN, S. (1986), "Religious Observance within a Human Capital Framework: Theory and Application", Applied Economics, n 18: 11, pp. 1193-202.

NEUMAN, S. y A. ZIDERMAN (1986), "How does Fertility relate to Religiosity: Survey Evidence from Israel”, Sociology and Social Research, $\mathrm{n}^{\circ} 70$, pp. 178-180.

OLDS, K. (1994), "Privatizing the Church: Diestablishment in Connecticut and Massachusetts", Journal of Political Economy, $\mathrm{n}^{\circ}$ 102: 2, pp. 277-97.

RASKOVICH, A. (1996), "You Shall Have No Other Gods Besides Me: A Legal-Economic Analysis of the Rise of Yahweh", Journal of Institutional and Theoretical Economics, $\mathrm{n}^{\circ} 152: 3$, pp. 449-71.

RODERO, J. y P. BRAÑAS-GARZA (2000), "Hotelling and the Olympus: Modelling Differences in Religious Prices", Central European Journal of Operation Research, $\mathrm{n}^{\circ}$ 8: 4, pp. 265-283.

RODERO, J., P. BRAÑAS-GARZA, A. GOURLAY y A. LORCA (2002) "Believe or not Believe: Generalising the Azzi-Ehrenberg Model”, Cuadernos de Economía, n 25: 1, pp. 33-44.

SACERDOTE, B. y E.L. GLAESER (2001), "Education and Religion", NBER working paper, $\mathrm{n}^{\circ}$ 8080 , January.

SAWKINS J. y I. PATERSON (1996), "An Occupational Analysis of Methodist Local Preachers in Scotland," mimeo, Department of Economics, Heriot-Wattt University, DP 96/18.

SMITH, A. ([1776]1965), An Inquiry into the Nature and Causes of the Wealth of Nations, Nueva York, Modern Library.

STARK, R. (1972), "The Economics of Piety: Religious Commitment and Social Class", Issues in Social Inequality, pp. 483-503, Boston, Little Brown.

STARK, R., L.R., IANNACCONE y R. FINKE (1996), "Religion, Science and Rationality", American Economic Review, $\mathrm{n}^{\circ}$ 86: 2, pp. 433-437.

STONEBRAKER, R. (1993), “Optimal Church Size: the bigger the better?”, Journal of the Scientific Study of Religion, $\mathrm{n}^{\circ}$ 32: 3, pp. 84-113.

SULLIVAN, D. H. (1985), "Simultaneous Determination of Church Contributions and Church Attendance", Economic Inquiry, n $23: 2$, pp. 309-20.

TAMURA, R. (1999), "Human Capital, Fertility and Economic Growth", Economy and Society, pp. 89-104, Cambridge, Cambridge University Press.

ULBRICH, H. y M. WALLACE (1983), "Church Attendance, Age, and Belief in the Afterlife: Some Additional Evidence", Atlantic Economic Journal, n' 11: 2, pp. 44-51. 
WALLIS, J.L. (1990), "Modelling Churches as Collective Action Groups", International Journal of Social Economics, $\mathrm{n}^{\circ}$ 17: 1, pp. 59-72.

WEBER, M. (1930), The Protestant Ethic and the Spirit of Capitalism [Traducción inglesa de Talcott Parson], Londres, Allen \& Unwin]. 